$\begin{array}{llllllll}\text { T } & \text { E } & \text { M } & \text { A } & \text { T } & \text { I } & \text { C } & \text { A }\end{array}$

\title{
ENFERMEDAD Y MEDICINA ENTRE LOS SHIPIBO-CONIBO DEL ALTO UCAYALI
}

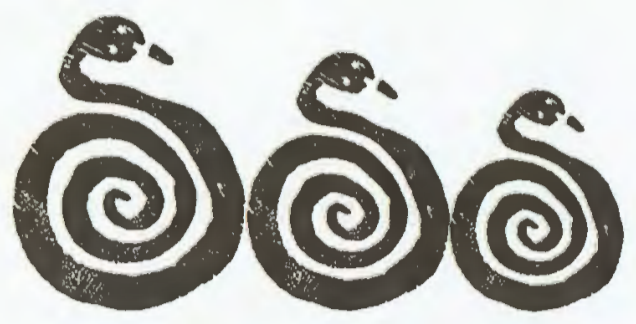

por J. Tournon; U. Reátegui

Ce travail est basé sur des enquetes portant a la fois sur l'Ethnomédecine et l'Epidemiologie que nous avons mené cher les Shipiba-Conibo du Hout Ucayali. Ce travail considére donc dे la fois le point de vue du malade, nous permel d'approcher ainsi les conceptions des Shipibo-Conibo sur la maladie (nosologie, ethiologie) et le point de vue du médecin, représenté par son dignostic, aidé d'analyses (bémoglobine, parasites). Nous examinons eussi les differents systémes thérapeutiques "iraditionnels" et "académiques" présents. Ces enquêtes ont eté réalisées dons deux communautés Shipibo-Conibo, une non inondable et l'autre inondable par l'Ucayali, des differences notables sont observées dans la fréquence des maladies de la peau.
We report on results of fieldwork done among the Shipiba-Conibo of the Upper Ucayali. The work included boib the notive's point of view, which allows us to approach the Shipibo. Conibo's of conceptions on bealth and disease (nosology, ethology) and included also the physician's point of view. The diferen' therapentic sysiems (traditional and academic) in wse in the Shipibo-Conibo communities are examined. The survey was done on the entive population ( 350 people) of two villages in two different environments: one can be flooded in the rain season and not the second. Significant differences are found between the two, in particular in the skin disease fregencies. 
Es werden im Artikel die Ergebnisse von Feldforschungen unter den Shipibo-Conibo vom Oberlauf des Ucayali dargelegt. Bei der Arbeit wurde neben der Perspektive des Arzles auch die einheimische Sichrweise mit einbezogen, die eine. Anndberung an die Shipibo-Conibo-Konzeptionen von Krankbeit und Gesundheit (Nosologie, Ethologie) erlaubt. Auch wurden dis unterschiedlichen therapeutischen Systeme (das traditionelle und das akademische). untersucht, die gegenwärtig in den Shipibo-Conibo-Dorfgemeinschaften praktizient werden. Bei der Untersuchung wurde die gesamie Bevolkerung zweier Dorfgemeinschaften in unterschiedlichen Umweitbedingungen erfasst: die eine kann in der Regenzeit überschwemmt werden, die andere nicht. Dabei stellten sich sigrifikante Unterschiede zwiscben beiden beraus, vor allem binsichtlich der Häufigkeit des Auftretens von Hautkrankbeiten.

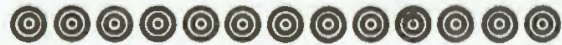

Estę artículo, sobre la enfermedad y la salud entre los Shipibo-Conibo, se dirige a todos los que se interesan en las culturas del Perú y de su selva, y en particular a los trabajadores de la salud en el Ucayali. Muchos de ellos, cuando no son Nativos, ignoran la existencia de un conjunto de concepciones y conocimientos vinculados con la salud, y con una cultura compleja que hay que tomar en cuenta para cualquier acción o política médica en la zona. De los Nativos esperamos comentarios y críticas.

Nuestros resultados sobre las enfermedades entre los Shipibo-Conibo son de dos índoles: tratamos en paralelo la epidemiología, desde el punto de vista del médico, y la etnomedicina, desde el punto de vista de los Nativos.

En el curso del presente trabajo nos referimos a las problemáticas tratadas por C.A. Seguín y sus colaboradores del Instituto Peruano de Psiquiatría Social en relación con la etnomedicina y la medicina folklórica en las distintas regiones del Perú (C.A. Seguín, 1979): costa, sierra, selva. H. Lumbreras C. dió un panorama de la compleja patología presente en la Selva Peruana (H. Lumbreras C., 1982). Varias encuestas epidemiológicas en la Selva Peruana han sido publicadas (E.A. Berlin, J. Soto, I. Temple) que tratan de otras poblaciones que los Shipibo-Conibo. La medicina y la terapéutica Shipibo-Conibo han sido estudiadas por G. Arévalo y A. Gebhardt-Sayer, sobre todo la función y el papel de los distintos curanderos (Onanya, Meraya) que utilizan los alucinógenos. Las terapias por el "raomis" y las plantas medicinales han sido documentadas por J. Tournon, U. Reátegui y col. $(1983,1984,1986)$. 


\section{A. METODOS}

Los resultados se basan sobre datos que provienen de una serie de encuestas hechas entre los años 1983 y 1986: encuestas estructuradas, es decir con preguntas fijas, y no estructuradas, en las cuales las preguntas pueden variar con el paciente.

La investigación siguió el curso siguiente:

$1^{\circ}$ - - Encuestas no estructuradas sobre nosografía y etiología nativas que se iniciaron con el estudio de las plantas medicinales y entrevistas del Doctor U. Reátegui con pacientes nativos en su consultorio. Estas encuestas nos permitieron preparar la encuesta estructurada.

$2^{\circ}$ - - Una encuesta estructurada conducida en Junio 1985 en dos comunidades nativas del Alto Ucayali, sobre una población de 320 nativos, con fichas conteniendo 70 items o preguntas. En este artículo analizamos sólo los items de carácter médico.

Estos items se pueden clasificar en dos partes (ver anexo):

- números 17 a 53: corresponden al punto de vista del paciente nativo (o de sus padres en el caso de niños), las entrevistas fueron anotadas y traducidas por el intérprete-traductor del equipo;

- números 54 a 68: corresponden al punto de vista de los investigadores: 54 , peso, y 55, talla, son datos antropométricos, 56 y 57 son datos de odontología, 58 hasta 66 son los resultados de análisis de las muestras de sangre y heces hechas en el laboratorio del Dr. U. Reátegui en Pucallpa, 67 y 68 son los diagnósticos que el Dr. U. Reátegui hizo primero en las CCNN (comunidades nativas) y después en Pucallpa con los datos de los análisis.

Para la encuesta estructurada escogimos las dos comunidades de Nuevo Nazareth y Puerto Nuevo por las razones siguientes:

- Nuevo Nazareth es de "bajeal", es decir inundable cuando crece el Ucayali, en cambio Puerto Nuevo es de "altura", no inundable;

- estas dos CCNN están a sólo unas horas con botes "peke peke";

- son de tamaño mediano, con poblaciones de 210 Nativos para Nuevo Nazareth y 114 para Puerto Nuevo, lo que nos permitió llevar a cabo las encuestas con los medios disponibles.

En cada comunidad se examinó toda la población presente y no sólo una muestra de esta población. Junto con la encuesta se atendió la salud de la población. El grupo de investigadores (médico, antropólogos, intérpretes) se ubicó cerca de la escuela y los habitantes vinieron casa por casa.

Las tomas de muestras de sangre y heces fueron hechas el último día de la investigación en cada comunidad y llevados directamente al laboratorio del Dr. U. Reátegui 
en Pucallpa, donde llegaron 36 horas después. A pesar de estas precauciones varias muestras de heces llegaron resecas a Pucallpa y no pudieron ser analizadas.

El estudio de la etnomedicina en comparación con el de la etnobotánica presenta nuevos problemas vinculados a su objeto mismo.

Las enfermedades no se pueden definir, aislar, manipular o conservar como las plantas. Como dice C.O. Frake (1961): "una enfermedad, p. ej. dolor de cabeza, no es algo que se puede designar con el dedo, tampoco dos ejemplos de una determinada enfermedad pueden ser puestos juntos para una comparación visual, y puestos en contraste como dos plantas". Es que una enfermedad no existe independiente del paciente. Así una enfermedad que tiene una misma causa o etiología, p. ej. un mismo agente infeccioso, puede evolucionar de manera diferente según el paciente. En cambio como dice este mismo autor: "una plantita de una determinada especie siempre crece y madura como una planta de la misma especie". Entonces no es sorprendente que la organización de los conocimientos médicos tomen una mayor complejidad que la de la etnobotánica u otros campos de la etnociencia.

El ambiente ecológico y los agentes infecciosos presentes son factores que determinan en gran parte las patologías de una población humana. Sin embargo son determinantes también los factores sociales y culturales: modos de alimentación, viviendas, uso del agua, disposición de los desechos...

Algunos antropólogos y psiquiatras declaran que las enfermedades dependen de la sociedad y aún más, que es la sociedad misma la que las crea. Esta declaración se puede entender de dos maneras:

1. Las enfermedades dependen del ambiente y de las condiciones de vida que la sociedad contribuye a crear. Citamos las patologías vinculadas con el uso de alcohol, tabaco, el consumo de ciertos alimentos, los accidentes ocupacionales...

2. Las representaciones de las patologías dependen de la cultura, de los niveles científicos y técnicos de la sociedad. Así una misma enfermedad podría ser considerada como causada por la posesión de un demonio, por el consumo de tal alimento, por un complejo de Edipo, una lesión o un desbalance bioquímico del sistema nervioso central.

En este trabajo tomamos como sistema de referencia la biomedicina o medicina académica. Sin embargo si el paradigma de la biomedicina puede ser considerado como universal, aceptado por todas las escuelas de medicina, todos sus aportes, métodos, medios humanos y técnicos no pueden ser considerados como universales, ya que no están al alcance de todos los médicos y pacientes. Por esto, en este capítulo, precisamos los medios biomédicos utilizados en la encuesta. 


\section{B. RESULTADOS}

La biomedicina distingue tres partes: nosología, etiología y terapéutica. Respetamos esta división para el análisis de la medicina nativa, tomando en cuenta que este trabajo se dirige en gran parte a los trabajadores de la salud.

\section{NOSOLOGIA}

La nosología se define como una parte de la medicina que trata de las enfermedades clasificándolas según criterios que permiten definir cada tipo de enfermedad. En este trabajo vamos a hablar de nosologías biomédica y nativa. Veremos que la nosología nativa es bastante compleja, a pesar de sus limitaciones.

Los datos sobre la nosología nativa provienen de dos tipos de fuentes:

1. Las encuestas lingüísticas en las cuales conseguimos términos en idioma Shipibo-Conibo que designan diferentes categorías de dolencias y enfermedades, de las cuales hicimos un análisis semántico.

2. La encuesta médica estructurada con fichas, que nos da para cada paciente, en idioma nativo, sus síntomas, el nombre de la enfermedad que padece (preguntas 20, 21) y también da los resultados de varios análisis (preguntas 58-60) y el diagnóstico médico (preguntas 67, 68).

\section{SIGNOS, SINTOMAS Y SINDROMES}

Entre los términos que describen o designan enfermedades la nosología biomédica distingue las categorías: signos, síntomas y síndromes.

Signos y síntomas.- Unas fuentes (Diccionario de Ciencias Médicas Dorland, 1981) definen el signo como una evidencia objetiva, el síntoma sería una evidencia subjetiva. Otras fuentes (Diccionario terminológico de ciencias médicas, L. Cardenal, 1958) consideran como síntomas ambos tipos de evidencias: las objetivas (evidentes a los sentidos del observador) y las subjetivas (percibidas únicamente por el enfermo). Es la definición que tomamos en lo siguiente donde consideramos sintomas como toda manifestación ostensible de enfermedad que sea objetiva o subjetiva. Es evidente que signos y síntomas van a cambiar de una población humana a otra, ya que dependen de las condiciones ambientales y de la cultura. 
Sindromes.- Citamos dos definiciones de las mismas fuentes:

- "conjunto de signos y síntomas que se presentan simultáneamente y caracterizan clínicamente un estado morboso, llamado también conjunto síntomático";

- "cuadro o conjunto sintomático, serie de síntomas y signos que existen a un tiempo y definen clínicamente un estado morboso determinado".

La historia de la biomedicina nos muestra que los síndromes cambian con los avances de la ciencia y de la tecnología médicas.

En este capítulo vamos a examinar como los Nativos Shipibo-Conibo organizan el campo semántico de la nosología y en particular si ellos distinguen también entre síntomas y sindromes.

Dentro de las fichas de nuestra encuesta estructurada (cf. anexo), la pregunta 20: ¿Cuáles son tus molestias?, se refiere más bien a los sîntomas. En cambio la pregunta 21: ¿Cómo se llama tu enfermedad? Es más destinada a revelar los síndromes.

Vamos a definir criterios operacionales que nos permitan distinguir entre estas respuestas las que corresponden a síntomas y síndromes. Varios casos se presentan:

$1^{\circ}$. - La respuesta a 21 es diferente de la 20. P. ej. encontramos unas fichas así:

20: "yoran chesshai, ponetei" (dolor de cuerpo, dolor al brazo),

21: "matsi jiquia"

En este caso consideramos que "yoran chesshai" y "ponetei" son síntomas y que "matsi jiquia" es un síndrome, conjunto de estos síntomas, y quizás de otros. "Matsi jiquia" corresponde a reumatismo como lo veremos abajo. En este caso se puede hablar de un síndrome nativo.

$2^{\circ}$ - - Dos o más términos se encuentran juntos en ambos items 20 y 21 con alta frecuencia. En este caso aún si los Nativos reconocen la asociación frecuente de varios síntomas, no se puede realmente hablar de un síndrome nativo.

Para estudiar los detalles de la nosología Shipibo-Conibo agrupamos las patologías en grandes categorías con los números de casos y los porcentajes de las poblaciones examinadas, Cuadro 1. Estas categorías son basadas sobre los diagnósticos del médico, preguntas 67 y 68 de las fichas. Sin embargo anotamos que a este nivel de clasificación hay muy pocas discrepancias entre el punto de vista del médico y el del paciente nativo. La sola excepción fue la de un paciente que se quejaba de estar "yosmairesin" (débil) por "chassho copia" (cutipado de venado), quien fue considerado como "chiquish" (perezoso) pero sano por el médico.

El Cuadro 1 nós enseña una situación sanitaria similar a otras ya observadas en poblaciones vecinas (E.A. Berlin, J. Soto, I. Temple) por la frecuencia de afecciones digestivas y respiratorias, sin embargo observamos muy pocos casos de paludismo y leishmaniasis. Es interesante notar que las afecciones de la piel son mucho más numerosas en la comunidad de Nuevo Nazareth que en la de Puerto Nuevo (ver abajo). 


\begin{tabular}{|lcccc|}
\hline \multicolumn{5}{c}{ Cuadro 1: N: números de casos, \%: porcentajes } \\
\hline \multicolumn{4}{c}{ Nuevo Nazareth } & Puerto Nuevo \\
& $N$ & $\%$ & $N$ & $\%$ \\
\hline sanos & 10 & 4.8 & 15 & 14 \\
Patologías: & & & & \\
digestiva & 109 & 53 & 45 & 42 \\
respiratorias & 14 & 6.8 & 10 & 10 \\
algias & 14 & 6.8 & 14 & 13 \\
piel & 34 & 16 & 3 & 3 \\
uro-genital & 7 & 3.4 & 1 & 1 \\
traumas & 6 & 2.5 & 2 & 2 \\
anemias & 4 & 2 & 2 & 2 \\
neurológicas & 4 & 2 & 2 & 2 \\
odontológicas & 3 & 1.5 & 3 & 3 \\
ceguera & 3 & 1.5 & 2 & 2 \\
otitis & 2 & 1 & 3 & 3 \\
gripe, paludismo & 2 & 1 & 2 & 2 \\
leishmaniasis & 1 & 0.5 & 0 & 0 \\
\hline
\end{tabular}

\section{PATOLOGIAS DIGESTIVAS}

Son las más comunes, ya que $53 \%$ de los habitantes de Nuevo Nazareth y $42 \%$ de los de Puerto Nuevo padecen de trastornos digestivos: enterocolitis y gastroenterocolitis. Estos son poli-infecciosos con un parasitismo casi de toda la población, ya que la infección con al menos uno de los tipos de helmintos que buscamos (items 59-62: ascaris, tricocefalo, uncinaria, strongyloides de las fichas), representa $93 \%$ de la población examinada en Nuevo Nazareth y $97 \%$ en Puerto Nuevo (Cuadro 2).

\begin{tabular}{|lcccc|}
\hline \multicolumn{4}{c|}{ Cuadro 2 } \\
\hline \\
\hline \multicolumn{4}{c|}{ Números de muestras } & \multicolumn{2}{l|}{ Porcentajes } \\
& con parásitos & sin parásitos & con & sin \\
\hline Nuevo Nazareth & 153 & 13 & 93 & 7 \\
Puerto Nuevo & 68 & 2 & 97 & 3 \\
\hline
\end{tabular}


Los sintomas nativos correspondientes más comunes son: "chisho" = diarrea, "quinanai" = vómito, "porocan chesshai" = dolor de barriga.

Otro síntoma es "estei" que parece designar un balonamiento abdominal o un abdomen globuloso demasiado frecuente entre los niños, llamados "buchizapas" en español local.

El término "noin", que designa a los gusanos, aparece en pocas fichas.

"Nihue bia": Este término se refiere a un conjunto de síntomas, "poro chesshai" = mal de estómago y "estei" = balonamiento de estómago o abonbado, y lo consideramos como un síndrome. También evoca una etiologia, pues "nihue bia" significa "traído por el viento". Parece que se hace un vínculo entre el aire del viento y el que está dentro del estómago. Se puede decir que este síndrome es llamado a la vez por su sintomatología y su etiología. Esta última puede tener varias causas, como lo veremos abajo.

\section{PATOLOGIAS RESPIRATORIAS}

En importancia vienen después de las digestivas. Varios pacientes ( 3 en Puerto Nuevo y 4 en Nuevo Nazareth) observados tenían evidencias de tuberculosis pulmonar y necesitaban ser examinados en Pucallpa.

Los síntomas nativos son:

"Ocoi" = tos, se reconoce dos tipos de tos: "oco manshanbicho" = tos seca y "oco michoquin" = tos con flema, de "michoquin" = botar; "jimi ocoi" es la tos con sangre o hemoptisis.

"Piniti" indica una bronquitis.

\section{ALGIAS}

Agrupamos en esta categoría varios tipos de dolores: neuralgias, mialgias y dolores osteo-articulares. Incluimos también en esta categoría los accidentes y sus secuelas.

Los síntomas correspondientes a la pregunta 20 de las fichas son: "yoran chesshai" = dolor de cuerpo, o por ejemplo: "ponetey, pecaten, quishin, huetasshon chesshai" = dolor de brazo, espalda, muslo, pierna, que designan a la parte del cuerpo que duele. 
En estos casos encontramos en la pregunta 21:

"matsi jiquia", que es claramente un síndrome nativo como ya lo vimos. Ya vimos en trabajos anteriores un buen número de "rao", plantas medicinales, para tratar "matsi jiquia".

Otros casos de "matsi jiquia" que salieron de la encuesta estructurada fueron: "baquenassh matsi jiquia" que designaba dolores después del parto, y "camosh natasha matsi jiquia" para secuelas de una picadura de chuchupe (herp., Lachesis muta).

Puesto que "matsi" significa frío, se puede preguntar si no existe: "sshana jiquia", "sshana" = frío, encontramos en la encuesta un solo caso de este síndrome. Los nativos distinguen los dos síndromes según la presencia de los síntomas siguientes: "chesshai" = dolor, "yona" = fiebre, "sshoa" = comezón, "soica" = hinchazón, como lo indicamos en el Cuadro siguiente:

Cuadro 3

\begin{tabular}{lcccc}
\hline & chesshai & yona & sshoa & soica \\
\hline $\begin{array}{l}\text { matsi jiquia } \\
\text { shana jiquia }\end{array}$ & + & - & - & - \\
\hline
\end{tabular}

Es el único caso de oposición frío/caliente que encontramos en la nosología shipiboconibo.

Encontramos otros síntomas que pertenecen a esta categoría: "misco" = calambre, "yossho" = embarramiento o músculos torcidos, "maban" = encogimiento de la rodilla, que no entran en el síndrome de "matsi jiquia".

Muchas de estas mialgias se pueden considerar como enfermedades ocupacionales, causadas por las cargas excesivas manipuladas y llevadas por los nativos: costales de productos de la chacra, motores...

\section{PIEL}

En el Cuadro 1 se ve claramente que las afecciones de la piel son más frecuentes en Nuevo Nazareth que en Puerto Nuevo: 34 y 3 casos respectivamente, o sea $13 \%$ y $3 \%$. Como ya lo explicamos en la Introducción estas dos CCNN tienen ambientes claramente distintos. Los factores ligados a esta diferencia que pueden explicar la mayor frecuencia de enfermedades de la piel en Nuevo Nazareth podrían ser: 
- La mayor abundancia de zancudos (ent. Anopheles spp.) y otros insectos;

- La diferencia de la calidad del agua disponible para lavarse y lavar la ropa en las dos CCNN. Puerto Nuevo se ubica directamente sobre el Ucayali donde la corriente es rápida y las aguas contaminadas se evacúan bien, en cambio Nuevo Nazareth se ubica sobre un brazo del Ucayali que está en proceso de ser colmado y donde la corriente es muy débil y casi inexistente en el verano, y tal vez no es suficiente para evacuar las aguas contaminadas.

En el Cuadro 4 se pueden observar con más detalles las enfermedades de la piel en estas dos CCNN. Dícho cuadro está compuesto de 9 categorías nativas de afecciones de la piel a las cuales tratamos de dar correspondencias biomédicas. Los términos nativos serán analizados abajo.

Cuadro 4

\begin{tabular}{lcc}
\hline & Nuevo Nazareth & Puerto Nuevo \\
\hline "sheno, yora payoti" & & \\
micosis sin o con sobreinfección & 20 & 1 \\
"jan payota", moniliasis & 2 & 0 \\
"nombe", tipo furonculosis & 3 & 0 \\
"cobeshe joconi", tipo impetigo & 4 & 0 \\
"tacho", tipo escamoso & 2 & 0 \\
"shate", herida infectada & 0 & 2 \\
"meno", quemadura & 1 & 0 \\
"sshinsshin", sarna & 1 & 0 \\
"repayoti", leishmaniasis & 1 & 0 \\
\hline TOTAL: números de casos & 34 & 3 \\
& $13 \%$ & $3 \%$
\end{tabular}

Se destaca la frecuencia de las micosis en Nuevo Nazareth. Los nativos se bañan en el río dos veces al día y no se les puede reprochar de falta de higiene. Pero como ya lo dijimos antes las aguas del Ucayali al nivel de Nuevo Nazareth pueden estar contaminadas, sin contar que por razones económicas los nativos utilizan poco los jabones.

Los Shipibo-Conibo tienen un vocabulario abundante para designar y escribir las afecciones dermatológicas, que tratamos de analizar en lo siguiente. C.O. Frake (1961) 
en un artículo clásico ya había mencionado la existencia de un vocabulario muy elaboI udo para las enfermedades de la piel entre los Subanum de Mindanao (Filipinas), que vlven también en un medio tropical húmedo. Esta convergencia se podría explicar por In frecuencia de dermitis en climas tropicales húmedos y también la fácil observación de las dermitis.

Una primera encuesta con informadores Shipibo-Conibo y un Atlas de Dermatologfa (G.M. Levene, C.D. Calman, 1974), nos permite conseguir los equivalentes de términos biomédicos de signos y sintomas:

mancha o mácula = "ssheno"

costra $=$ "shaca" (significa también corteza de ärbol)

pápula $=$ "nombe"

módula = "ani nombe"

pústula = "huacho" o "coo nombe" ("coo" = pus)

quiste = "jobo" o "huaca bero" (vaca/ojo)

ampolla o vesícula = "tossh bata" o "tosshba", que "no es una enfermedad pero se produce en el pie por caminar mucho o en la mano por el machete"

corte $=$ "shate"

úlcera = "peque", cuando la úlcera se extiende se llama "pequeta".

Los Shipibo-Conibo no tiene un término único para designar las micosis. A sus primeras etapas se utiliza el término "ssheno" (mancha). Se distinguen el "jossho ssheno" (blanco/ssheno), el "sshoa ssheno" (comezón/ssheno). El "jossho ssheno" puede dar el "joshin ssheno" cuando hay una eritema.

Los nativos atribuyen los "ssheno" a la ingestión de ciertas carnes, de pescados como el "ipo", carachama (Pterigoplichthys multiradius), o de mamíferos como "ahua", sachavaca o tapir (Tapirus terrestris), el "sapen" o vaca marina (Trichechus inunguis) o el "cochi", puerco. Casos de "ssheno" son atribuidos al hecho de no respetar una dieta después de la ingestión de ciertos "rao", como el "moca pari", chiri sanango (Brunfelsia grandiflora).

Cuando la micosis se extiende y se sobreinfecta con bacterias, se utiliza el término "payoti" (que puede significar en otros contextos: podrido) en composición con la primera sílaba del término que designa la parte afectada:

"yora payoti" o "rapayoti" de cuerpo/payoti,

"mapayoti" de "mapo" = cabeza,

"tapayoti" de "tae" = pie,

"mepayoti" de "mequen" = mano,

"janpayoti" o "jan payota" de "jana" = lengua, designa a la moniliasis,

"repayoyi" de "requin" = nariz, designa a la uta o leishmaniasis forma espundia,

El "sshinsshin" designa sin ambigüedad a la sarna, causada por acaros.

Se ve que los Shipibo-Conibo perciben y nombran bien los diferentes aspectos que presentan las enfermedades de la piel. Sin embargo la nosografía nativa no sigue 
exactamente la biomédica, por ejemplo una determinada entidad nosográfica biomédica como "micosis" puede ser designada en idioma shipibo-conibo por varios términos según su evolución: "jossho ssheno", "josshin ssheno", "payoti”, Pero los Nativos saben que los "ssheno" pueden evolucionar en "payoti".

\section{ETIOLOGIA}

Con el estudio de la etiología nativa entramos aun más en la visión que el Shipibo-Conibo tiene de su medio ambiente o "ecovisión" (término que preferimos al término de "cosmovisión"). El medio ambiente amazónico es especialmente abundante en especies animales y vegetales que puede explotar el habitante del Ucayali. Sin embargo muchas de estas especies contienen toxinas en pequeñas o grandes concentraciones que pueden ser factores etiológicos.

En el caso de la Etiología como en el de la Nosología, las encuestas nos dan tanto el punto de vista del nativo que el del médico.

El estudio de las etiologías ha inspirado varios trabajos teóricos a los cuales nos referimos (G.M. Foster). Se pueden distinguito dos clases de etiologias:

1. Etiologías personalizadas, en las cuales el agente etiológico es personalizado, ya sea una persona, un animal, una planta, un dios, un espíritu.

2. Etiologías naturales, que representan los factores etiológicos en términos impersonales o sistémicos: frí, calor, viento, humedad o como desbalances en estos elementos.

La distinción entre los dos tipos no es siempre obvia. Por ejemplo A. Seguín, M. Chiappe, C.M. Dobkins del Río, 1979, observaron que para un mismo síndrome como el "daño", los agentes etiológicos pueden ser personas o fuerzas impersonales y mostraron que cambian con las condiciones ambientales y sociales. Vamos a dar también ejemplos de los Shipibo-Conibo.

Con las etiologias personales se pueden encontrar varios niveles de causalidad (G.M. Foster):

- las causas eficiente e instrumental, p. ej. en el caso de un "brujo" nativo acusado de haber enfermado a un paciente con una espina de chonta o virote de chonta (bot., cf. Bactris sp., Palmacea), el "brujo" sería la causa eficiente y la espina la causa instrumental. A veces entra una tercera causa, "causa final", que trata de contestar a la pregunta: ¿Por qué esta enfermedad me ocurrió a mí? Veremos más ejemplos de esto en la etiología shipibo-conibo.

En las fichas de la encuesta estructurada (cf. anexo), las preguntas 23 hasta 42 están destinadas a conocer la etiología nativa. Las preguntas 23 y 24 son para saber si 
lus Shipibo-Conibo conocen el concepto biomédico de microbio. Las preguntas 25 hunta 30 son para conocer las percepciones etiológicas: ¿Qué viste, tocaste, tomaste, uliste, soñaste que te enfermó? Las preguntas 31 hasta 42 son más especiales y destinadas a investigar el concepto Shipibo-Conibo de "copia", que corresponde al concepto de "cutipado" en español local (ver abajo).

A la pregunta: ¿Viste algo que te enfermó?, encontramos las respuestas: "mahua" - muerto. Ver un muerto es una causa frecuente de enfermedad, sobre todo para niños.

¿Qué comiste, tomaste que te enfermó? Vemos que la ingestión de "mai" = tierra, o geofagia, por los niños es frecuente (once casos) y es considerada como una crusa de gastroenterocolitis. En los casos correspondientes los análisis de las heces mostraron una abundancia de paråsitos: ascaris, tricocefalos, uncinarias. Un patrón de esta geofagia es que se encuentra entre los niños de un número limitado de familias. lïstos niños se llaman "buchizapas" en español local.

La ingestión de carnes de ciertos animales o de ciertas plantas medicinales o "rao" es una causa frecuente de enfermedad, ver abajo.

"COPIA", el cutipado

El concepto de "copia" juega un papel muy importante en la etiología shipiboconibo. Se puede traducir en español local por "cutipado". El Vocabulario del Oriente Peruano de E.D. Tovar, 1966, nos da: cutipar = hacer maleficio. Supimos de "copia" por primera vez en nuestra encuesta sobre los "rao" (J. Tournon, 1986). Unas plantas medicinales necesitan seguir una dieta sino pueden cutipar, lo que se concretiza en una enfermedad. Esta creencia es vinculada con el carácter ambivalente de los "rao" que tienen una fuerza, positiva para sanar o negativa para dañar. Así anotamos: "huapan joni copia riqui" = el "huapan" ha cutipado a la persona, "huacan ehua baque copia riqui" = el saltón (icht. ver abajo) cutipó al niño, "moatian apaonique jato sshono copiqui ramamoa non oinyama, ramatian bicho iqui neino ja sshonomeran tetai jato jaconmai" = en este tiempo las lupunas nos podían cutipar, ahora son sólo las nutrias que nos pueden dañar por medio de las lupunas":

los lupunas serían la causa instrumental y las nutrias la causa eficiente,

"yoshin cosshqui copimis" = el arco iris puede cutipar,

"Imahua caya copia" = el alma del muerto le cutipó,

"jimi copia" = la sangre le cutipó.

Con estos ejemplos vemos una variedad de agentes que pueden "copia": un "rao", el "huapan" (planta colectada pero no identificada todavía), que se toma como medicina, un pescado, el saltón, que se comió, un árboll, la lupuna (cf: Chorisia insig- 
nis HBK), al lado del cual se ha caminado, un fenómeno atmosférico, el arco iris, el alma de un muerto, la sangre de una parturienta o de una menstruante.

Para saber más sobre "copia", le consagramos las preguntas 31 hasta 42 de la encuesta, a las cuales corresponden varios casos de pacientes cutipados por "rao". Un paciente tomó "cana chiari" = toe (Datura sp.) para ver quien le había robado, pero no había seguido su dieta y el toe le cutipó: en este caso el no respeto de la dieta por el paciente sería la "causa final". Otro caso fue el de un niño a quien su padre.dio "mansshamah cahuati" (Myrsinacea no identificada), que es un "mechati rao", para que tuviera éxito en la pesca y la caza. Sin embargo en el momento presente el niño está padeciendo lo que llamamos los "efectos secundarios" de este "rao". Encontramos unos "huaste" (Cyperus spp.) y un sanango (Brunfelsia sp. o Bonafousia sp., J. Tournon 1986) como "rao" etiológicos. Vemos unos pacientes que atribuyeron sus enterocolitis a los "chantomarin". Son algas (no identificadas) de color rojo oscuro que se ven en los charcos de agua que se quedan en las playas del Ucayali durante la estación seca.

Es importante notar que algunos árboles, considerados como "rao", pueden cutipar a distancia sin ingestión o contacto directo. Es el caso del "sshono", la lupuna (cf. Chorisia insignis), del "ana", catahua (Hura crepitans), del "ino shatan", aya huma (Couroupita sp.). Los Shipibo-Conibo dicen: "jihui yoshin copia" = los espíritus de los árboles cutipan.

En el curso de la encuesta encontramos el término nativo "merati" de significado muy semejante al de "copia", que también se puede traducir en español local por "cutipar":

"huapanma mera joni" = el "huapan" cutipó a la persona.

Sin embargo esta voz se puede encontrar en otro sentido:

"onanyani min amatiqui nishishea meran" = el "onanya" te puede sanar tomando el ayahuasca.

El verbo "merati" puede tener otros significados como: encontrar, enamorarse. "Mera" entra en otros términos como "meraya" que designa a una categoría de curandero (G. Arévalo, 1986) y "meramis" que califica a ciertos "rao" (J. Tournon et al., 1986) que tienen el poder ambivalente de dañar y curar. Esta ambivalencia nos parece diferenciar los dos términos "copiati" y "merati".

\section{ANIMALES ETIOLOGICOS}

Varios nombres de animales aparecieron'en la encuesta como factores etiológicos, saliendo de las preguntas:

25: ¿Qué viste que te enfermó?

27: ¿Qué comiste que te enfermó?

29: ¿Qué oliste que te enfermó?

31: ¿Hay algo que te cutipó? 
Los tipos de respuestas nos permiten clasificar los animales etiológicos en dos clases:

\section{Animales que enferman sin que sean comidos}

El "ronin", yacumana, boa gigante mítico, claramente no puede ser comido pero si puede enfermar cuando entra en los sueños de los Shipibo-Conibo.

Los animales no imaginarios que encontramos en la encuesta son:

"ochiti", el perro,

"coshoshca", el delfin, bufeo en español local (Sotaria fluviatilis),

"cape", el caiman, lagarto en español local (Caiman scletops),

"neino", nutria, lobo marino en español local (Pteronura brasiliensis).

El perro nunca se come entre los Shipibo-Conibo, pues puede cutipar, encontramos un caso en el cual fue acusado de haber producido una hernia. cutipa:

Se dice que el bufeo enferma por su aliento apestoso: "coshoshca pisi", también "coshoshca nihue biashon noa jaconmatiqui quinanti, chichoti, jaconma acaiqui, estei": los bufeos por su mal aliento nos hacen mal, nos dan vómito, diarrea, inflamación de barriga. "Nihue bia" (ver arriba) es un ejemplo de sindrome nativo que puede tener diferentes causas según los informadores. Se dice que se produce cuando hay un remolino de aire, "maya nihue", de estos muy frecuentes en el verano. Entonces el padre va a decir a su hijo no cruzar el remolino: "nihue min bechinaque", según G.M. Foster sería una causa natural. Pero puede tener una causa personalizada, siendo producido por el mal aliento del bufeo, y este mismo animal es a menudo considerado como un brujo metamorfoseado. También unos nativos nos contaron que los bufeos dañan por medio de los "tsitsimita", caneros (icht. Vandellis plazaii, especie de pescado parásito que puede entrar en los orificios), que mandan para atacar las personas que entran en el agua. En la Selva Peruana existen muchos cuentos sobre los bufeos, tanto entre los nativos como entre los mestizos. (U. Reátegui, 1983), a los cuales son atribuidos síndromes psicológicos. En la encuesta los bufeos habían producido gastoenterocolitis.

El "cape", caimán, también puede enfermar por su olor apestoso, "cape pisi". Había producido una mialgia y una micosis.

La nutria, "neino", juega un papel importante como factor etiológico. Al respecto A. Gebhardt-Sayer (1986) ha observado que tanto el perro, como el bufeo y la nutria son despreciados por los Shipibo-Conibo por no tener una época definida de celo. Encontramos una paciente quien se quejaba de dolores musculares por haber sido golpeada por una nutria, "neino retecana". Pretendia que la nutria le había mandado un "malefićio, "neino yotoa", y había consultado con un brujo, "yobe". El diagnóstico biomédico indicó un buen estado general. Encontramos otro caso de un paciente sufriendo de dolores de espalda, el espíritu de la nutria, "neino yoshin", le había cutipado y había consultado a un "onanya". 


\section{Animales que enferman por ser comidos}

La encuesta nos dió un buen número de animales correspondiente a esta categoría.

Entre los mamíferos encontramos:

"cochi", puerco doméstico,

"amen", ronsoco (Hydrochaerus hydrochaeris),

"chassho", venado (Mazama spp.),

"jono", sajino (Tayassu tajassu),

"joshin shino", mono colorado (no identificado).

Comer estos mamíferos produciría gastroenterocolitis. También observamos el caso de un paciente para el cual el consumo de veriado habia producido "chiquish", pereza. Entre los reptiles encontramos:

"cabori", taricaya (Podocnemis unifilis),

"copitso", charapa (Podocnemis expansa),

"manashahue", motelo (Geochelone denticulata),

"rono ehua", anaconda (Eunectes murinus).

El consumo de estos reptiles puede producir una variedad de enfermedades, sobre todo algias, pero también micosis y gastroenterocolitis.

Los pescados que se encuentran con más frecuencia como factores etiológicos, siempre son acusados de enterocolitis y gastroenterocolmitis:

"maque", pirañas (Serrasalmus spp.),

"ino banhuin", zungato (cf. Pseudopimelodus zungaro),

"shahuan huaran", pez torre (cf. Phractocephalus hemiliopterus),

"huacan ehua", saltón,

"charan ehua", achacubo.

Hay que notar que aparte de los pirañas, todos son silúricos.

Entre los invertebrados encontramos sólo el "nocho", churo, que es ụn caracol terrestre.

Para resumir podemos clasificar los animales etiológicos así:

1. Animales que enferman sin ser ingeridos:

a) Son comidos por otras poblaciones diferentes de los Shipibo-Conibo y pueden ser cónsiderados como comestibles: caimán, delfín.

b) No son comidos ni por los Shipibo-Conibo ni por otras poblaciones de la Amazonía Peruana: perro, nutria, no los consideramos como comestibles. 
2. Animales que enferman por ingestión: mamíferos, reptiles, pescados, un caracol terrestre.

Nos parece que entre los Shipibo-Conibo ciertos animales pueden ser etiológicos de maneras muy distintas.

Las interdicciones alimenticias, o tabús en el sentido amplio, es un tema clásico de investigaciones y especulaciones por parte de antropólogos, y varias teorías han sido elaboradas. Siguiendo E.B. Ross (1978), distinguimos por un lado las teorías "mentalistas": los animales prohibidos serían los que no entran en una taxonomía. Por otro lado encontramos teorías "materialistas" que tratan de explicar las prohibiciones alimenticias por razones ecológicas:

- los animales prohibidos no valen la pena cazar,

- los animales prohibidos son los que se pueden extinguir fácilmente por sobrematanzas, ya que su reproducción no es muy rápida, así la prohibición sería una manera de preservar la especie.

Sin embargo no creemos que todas las creencias etiológicas y prohibiciones alimenticias, en relación con animales de los Shipibo-Conibo puedan ser consideradas en un mismo plano ni explicadas por una sola teoría. Imaginamos un Nativo del Ucayali que llegaría a Lima, él encontraría tambié ‘ animales etiológicos: perros rabiosos o no rabiosos, arañas ponzoñosas, ratas y murciélagos que pueden asustar a personas nerviosas. El encontraría también un número de interdicciones alimenticias, p. ej. las fresas que pueden dar alergias y comezones, los pescados que no hay que dar a las personas que tienen una infección, carne roja que no hay que comer durante la semana santa. Opinamos que sería muy criticable cualquier intento de explicar todas estas interdicciones por una teoría única, ya que tienen orígenes distintos y determinan comportamientos distintos.

Los animales que nos parecen ser realmente prohibidos o "tabús" entre los Shipibo-Conibo son pocos, son los que corresponden a la categoría "1-a" de nuestra clasificación: el caimán y el delfín. Para el delfín una explicación sería la dificultad de su caza con las armas disponibles, para el caimán no vemos una razón y es consumido por grupos vecinos de economía semejante. Los mamíferos: venado, sajino, ronsoco, parecen estar prohibidos en algunos grupos de la Amazonía (E.B. Ross). Entre los Shipibo-Conibo son consumidos y a veces acusados de gastroenterocolitis.

A las explicaciones "materialistas" que propone E.B. Ross, podemos agregar otra que sería una toxicidad de la carne de ciertos animales, al menos en ciertas épocas. No es imposible si se considera que muchas especies vegetales comunes en la zona tienen toxicidad. P. ej. el Doctor U. Reátegui observó varios casos de intoxicación por consumo de carne de ronsoco. El consumo de los hígados de estos animales han producido hasta intoxicaciones graves, son los hígados que acumulan las toxinas. Es conocida la toxicidad de pescados perteneciendo a ciertas familias (M. Barreda O., 1978), sin embargo faltan evidencias en el caso de los silúricos como el saltón. 


\section{OLORES}

En un trabajo sobre la terapia del "meraya" y del "onanya", A. Gebhardt-Sayer, 1985, señala la importancia de los olores en la terapéutica shipibo-conibo. Plantas perfumadas, "inin rao", como el "arabaha" (Ocimum americanum) o el "huiroro" (Ocimum micranthum) son utilizadas por el curandero durante su sesión con el ayahuasca. Por otro lado los malos olores, como "pisi" y "huia", atraen los espíritus dañinos. En nuestra encuesta encontramos varios olores como factores etiológicos:

"pisi", p. ej. "cape, coshoshca pisi" = el mal olor del lagarto, del bufeo.

Se dice también "pisi queras" = el mal olor de la basura.

"huia", es el olor del pescado no cocido, fue acusado de casos de gastroenterocolitis, "poro isin".

"janssho", "jimi janssho", el olor de la sangre, especialmente de la sangre de una parturienta o de una menstruante, fue acusado de casos de gastroenterocolitis. Una paciente partera, "baquemis", atendió un parto estando en dieta por el "onanya", el olor de la sangre de la parturienta le hizo "torcer" su dieta, desde ahí ha vuelto a enfermarse con diarreas y dolor de estómago.

Es interesante notar que el vocabulario shipibo-conibo es abundante en términos designando olores, si anotamos otros términos:

"inin", olor de un perfume,

"itsa", olor del sudor, de un pescado o de una carne asada,

"nobe", olor de una rica cocina, puede significar también sabroso.

\section{TERAPEUTICA}

Dos sistemas terapéuticos están presentes en las Comunidades Shipibo-Conibo:

1. El sistema tradicional, o etnomedicina, arraigado en la cultura shipibo-conibo.

2. El sistema médico oficial o académico, que entra más y más en la sociedad nativa pero en forma a veces desfigurada como lo veremos.

Con las preguntas 44 hasta 53 de la encuesta tratamos de evaluar la importancia de cada uno de estos dos sistemas en las comunidades de Nuevo Nazareth y Puerto Nuevo, por el número de pacientes que consultan los diferentes agentes de los dos sistemas de salud.

3. El primer sistema es complejo y ya ha sido en parte descrito (G. Arévalo, A. Gebhardt-Sayer, A. Hansson, U. Reátegui, J. Tournon et al.). Se puede distinguir dos aspectos:

Por una parte se encuentra la utilización casera de los "rao", sobre todo vegetales, por los "raomis" (conocedores de medicinas). Otros agentes de esta medicina casera son los "tobiamis", de "tobi" = lisiado, que pueden ser considerados como kinesiterapeutas, y los "baquemis", de "baque" = criatura, quienes son las parteras. 
Cuadro 5

\begin{tabular}{lcccc}
\hline & \multicolumn{2}{c}{ Números de casos } & \multicolumn{2}{c}{ Porcentajes \% } \\
& Nuevo Nazareth & Puerto Nuevo & Nuevo Nazareth & Puerto Nuevo \\
\hline nadie & 44 & 23 & 25 & 25 \\
médico & 7 & 10 & 4 & 12 \\
promotor & 51 & 19 & 28 & 22 \\
total acad & 58 & 29 & 32 & 33 \\
raomis & 37 & 16 & 21 & 18 \\
tobiamis & 1 & 0 & 0,5 & 0 \\
baquemis & 0 & 0 & 0 & 0 \\
onanya & 36 & 15 & 20 & 17 \\
meraya & 1 & 1 & 0,5 & 3 \\
yobe & 1 & 3 & 0,5 & 39 \\
total trad & 76 & 35 & 42,5 & \\
\hline
\end{tabular}

Por otra parte se encuentran los "onanya", "meraya" y "yobe". Los dos primeros son "curanderos" y el tercero es más bien "brujo", experto en el envío de espinas, "yobe" en shipibo-conibo ("camori" en quechua) de chonta (ver arriba).

El Cuadro 5 nos da el número de pacientes que consultaron los diferentes agentes, $o$ a nadie.

Se ve adentro de esta medicina tradicional la preponderancia de los "raomis" y "onanya". En cambio las consultaciones con los "tobiamis", "baquemis", "meraya" y "yobe" son escasas. Sin embargo hay que notar que las funciones de "onanya" y "meraya" parecen poco diferenciadas, observamos que los nativos pueden nombrar a los mismos individuos indistintamente "onanya" o "meraya". El término "meraya" parece ser más prestigioso que el de "onanya". Sin embargo G. Arévalo dice que las dos funciones son bien distinguidas por los especialistas mismos. En cuanto al "yobe" es posible que sea más consultado de lo que muestra la encuesta ya que tiene un carácter esotérico y peligroso.

La pregunta 51 es destinada a conocer los "rao" que prescribe el "raomis". Encontramos "rao" vegetales ya vistos anteriormente (J. Tournon, 1986):

"pish pish", Aristolochia sp.,

"au", Crataeva tapia,

"sshoro", Carapa sp.,

"cana chiari", Datura sp.,

"paico", Chenopodium ambrosioides,

"boens", Pseudocalymna alliaceum,

"nane", Genipa americana, 
"marava", Malachra capitata,

"huiroro", Ocimum americanum,

"pionmis", Jatropha curcas.

Muchas de estas especies tienen actividad farmacológica.

Es importante notar que ahora los "raomis" utilizan también productos comerciales, desgraciadamente algunos de ellos no son medicinas y pueden ser peligrosos. Con la encuesta encontramos: agua florida, kerosene, limón con aceite de motosierra, petróleo, y aun peor Baygón (el insecticida bien conocido) para una bronquitis. En este caso, según C.A. Seguín (1979), no se trata de medicina tradicional o etnomedicina sino de medicina folklórica.

La encuesta nos enseña que los "raomis" pueden prescribit también medicinas comerciales: alcanfor, antalgina.

2. El sistema médico oficial se compone de los médicos, sanitarios y promotores de salud.

A pesar de las limitaciones que tiene la medicina occidental en el Ucayali, hay que reconocer el enorme progreso logrado por las vacunaciones de los niños que han permitido limitar en gran parte las epidemias que causaban tantas víctimas hasta hace pocas decenas.

En la época de la encuesta no había médicos permanentes en el Distrito de Iparia, donde se ubican las comunidades de Nuevo Nazareth y Puerto Nuevo. La consultación con médicos se hace en Pucallpa o por la visita de médicos en las CCNN. Las únicas consultas que supimos fueron del doctor U. Reátegui en Julio 1982 y la de la encuesta en Junio 1985. El Cuadro 5 nos muestra que el médico es muy poco consultado, $11 \%$ de los pacientes habían visto a un médico. Sin embargo esta cifra puede ser subestimada, ya que una fracción importante de los habitantes de las CCNN ausentes en el momento de la encuesta estaban de viaje en Pucallpa precisamente para consultar médicos. Si se supone que todos los nativos ausentes se habían ido a Pucallpa para consultar un médico, se puede estimar un límite superior de $16 \%$ de nativos que consultaron médicos, lo que también es poco.

Si ahora queremos comparar los porcentajes de pacientes que van a consultar el sistema tradicional o el sistema académico, la encuesta da una ligera ventaja al primero: $39 \%$ vs. $33 \%$ en Puerto Nuevo y $42 \%$ vs. $32 \%$ en Nuevo Nazareth (Cuadro 5 ). Vemos también que un pequeño porcentaje de pacientes se fueron a consultar los dos sistemas: $3.5 \%$ en Puerto Nuevo y $3 \%$ en Nuevo Nazareth.

La pregunta 51 está destinada a conocer las medicinas que prescribe el promotor de salud. Es interesante notar que el sanitario prescribe unos "rao" vegetales, como "rono huaste", Cyperus sp., para una picadura de serpiente. Un número de pacientes no podían precisar el nombre de la medicina prescrita y mencionaron sólo: "noin rao" (medicina para lombriz) o pastillas. Felizmente en muchos casos los pacientes se acordaron del nombre comercial de la medicina. 
El uso de anti-helminticos comerciales parece muy escaso: 3 casos en Puerto Nuevo y 1 en Nuevo Nazareth (mintezol), a pesar que cerca de $95 \%$ de las muestras de heces contenían parásitos (Cuadro 2). Observamos el uso frecuente del "paico" (Chenopodium ambrosioides), anti-helmintico de actividad comprobada, prescrito por el "raomis". Pero no observamos el uso del latex del oje, "sshomi" (Ficus glabrata). El uso de este latex sería mucho más apropriado a la realidad local que el del mintezol, muy eficaz pero relativamente caro y con serios efectos secundarios. El programa AMETRA (A. Hansson, 1986) estudia este latex y organiza programas de divulgación, desafortunadamente no había llegado a estas comunidades todavía.

Nos parece importante notar sobre la prescripción inadecuada de medicinas (ver la encuesta de J. Lasselain en el Cuzco). Así se prescribieron penicilina y terramicina para pacientes con enterocolitis y una carga elevada de helmintos, streptomicina para una persona que no tenía síntomas de tuberculosis. Vimos hasta un caso de enterocolitis con diarrea tratado con el "norfor", producto para los cánceres de los senos y del endómetro, y un caso de uso de "cerobran" para un niño de 11 años, infestado por ascaris y con cólicos y diarreas fuertes. La mayoría de las demás medicinas prescritas tratan más los síntomas que las causas: antalgina, mejoral, novalgina, sibalgina. En cambio observamos el uso de varias vitaminas que no puede ser sino positivo.

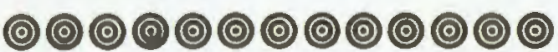

\section{CONCLUSIONES}

Estas encuestas médicas toman en cuenta ambos puntos de vista: el del médico y el del paciente nativo. Así nos dan datos a la vez de epidemiología y de etnomedicina. Los datos de epidemiología nos muestran un cuadro ya observado en otras zonas de la Amazonía, con la prevalencia de patologías digestivas y respiratorias.

En cuanto a la etnomedicina de los Shipibo-Conibo es muy compleja y vemos muchos saberes y concepciones sobre la salud y la medicina. Sin embargo nos parece a nosotros, con nuestra cultura biomédica, desigualmente desarrollada. Ciertas partes de la nosografía como la de las enfermedades de la piel son muy desarrolladas, con muchos términos, en contraste otras como la de las enfermedades digestivas nos aparecen más simples. Es interesante notar que los nativos tienen términos que designan a síntomas y otros a síndromes. La etiología shipibo-conibo nos hace acceder a todo un mundo de saberes y creencias sobre su medio ambiente visto como fuente de enfermedades. Las concepciones shipibo-conibo parecen tener poco en común con las de la Costa y las, de la Sierra Peruanas con su prevalencia del "susto" y del "daño", además vimos un sólo caso de oposición frí/cálido que parece estructurar las nosografías en la Costa y la Sierra. En cuanto a la terapéutica se destacan la coexistencia de dos sistemas: el tradicional o étnico y el académico o comercial. Pero los nativos todavía ven sino un débil reflejo de lo que les podría ofrecer este último. 


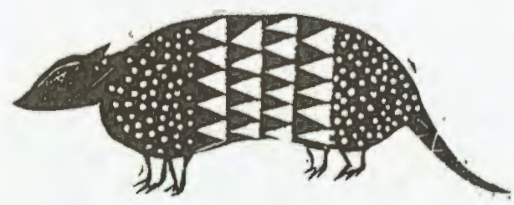

\section{B I B L I O G R A F I A}

AREVALO, G.V.

1986 El Ayahuasca y el curandero Shipibo Conibo del Ucayali, América Indigena, 46(1), 147.162.

BARREDA, 0 .

1978 Ictiología general, Lima.

BEHRENS, C.A.

1986 Shipibo Food Categorizations and Preference: Relationships between Indigenous and Western Dietary Concepts, American Anthropologist, 88, 647-657.

BERLIN, E.A.

1982 Parásitos y nutrición: Dinámica de la salud entre los Aguaruna-Jivaro, Amazonía Peruana, 3(6), 51-58.

BRADFIELD, R.B; LAURIAULT, J.

1961 Diet and Food Beliefs of Peruvian Jungle Tribes, J. of the American Dietetic Association, 39, 126-128.

Diccionario de Ciencias Médicas Dorland, 7a Ed., Editorial El Ateneo S.A., impreso en España 1981.

Diccionario terminológico de Ciencias Médicas, 6a Ed., por L. Cardenal, Salvat Editores, impreso en España.

FABREGA, $\mathrm{H}$.

1972 Medical Anthropology. En Biennial Review of Anthropology, 167-229.

FOSTER, G.M.

1976 American Anthropologist, 78, 773-782.

FRAKE, C.O.

1961 American Anthropologist, 63, 113-132.

GEBHARDT-SAYER, A.

1986 Una terapia estética. Los díseños visionarios del Ayahuasca entre los ShipiboConibo, América Indigena, 46(1), 189-218.

HANSSON,A. et al.

1986 Preclinical and Clinical Studies with Latex from Ficus glabrata HBK, a traditional intestinal anthelminthic in the Amazonian Area, J. of Ethnopharmacology, 17, 105 . 138.
LASSELAIN, J.

1987 Médicaments inessentiels, L'Harmattan, Paris.

LEVENE, G.M.; CALMAN, C.D.

1974 A Color Atlas of Dermatology, Wolfe Me dical Publications.

LUMBRERAS, H.C.

1982 Vista Panorámica sobre la Patología Peruana y Especial Referencia a aquellas enfermedades que requieren estudio, Amazonía Peruana, 3(6), 7-12.

PERRIN, $M$.

1980 Un succes bien relatif: la medecine occidentale chez les Indiens Guajiro, Soc. Sci. and Medicine, 14B, 279-287.

REATEGUI, U.

1983 Cushushca Yushin, Bufeo Colorado, Ed. Kemoy, Chiclayo.

ROSS, E.B.

1978 Food Taboos, Diet and Hunting Strategy: The Adaptation to Animals in Amazon Cultural Ecology, Current Anthropology, 19(1), 1-34.

SEGUIN, C.A.

1979 Psiquiatrí Folklórica, Ed. Ermar, Lima, SOTO, J. $156 \mathrm{p}$.

1982 Ecologia de la Salud en Comunidades Nativas de la Amazonía Peruana, Amazonía Peruana, 3(6), 13-26.

TEMPLE, I.

1978 Informe médico sobre una Comunidad Amuesha, en Salud y nutrición en Sociedades Nativas, A. Chirif, Compilador, CIPA, Lima.

TOURNON et al.

1986 Plantas y Arboles Medicinales de los Conibo del Alto Ucayali: Concepciones Nativas y Botánica, Revista Forestal del Perú, 13(2).

TOVAR, E.D.

1966 Vocabulario del Oriente Peruano, Universidad Nacional Mayor de San Marcos, Lima. 


\section{ANEXO: Ficha de encuesta}

20. Cuáles son tus molestias? Jahuen qui mia tseshacatai?

21. ¿Cómo se llama tu enfermedad? ¿Min isin qui jahue jane iqui?

23. ¿Tienes microbios?

24. ¿Quién te contagio?

25. ¿Viste algo que te enfermó? Jahue oinasca mia isin naiqui?

26. ¿Toqaste algo que te enferm 6 ? Jahue tiiacashca mia isinnaiqui?

27. ¿Comiste algo que te enfermo $\delta$ JJahue piashca mia isinnaiqui?

28. ¿Tomaste algo que te enfermó? Jahue sheashca mia isinnaiqui?

29. ¿Oliste algo que te enfermó? Jahue sheteashca mia isinnaiqui?

30. ¿Soñaste algo que te enfermó? Jahuequesca min namataiqui isinosh?

31. ¿Algo te cutipó? Jahuenca mia copia iqui?

32. ¿Reo yoshin?

33. ¿Rao meramis?

34. ¿Roo meramisma?

35. Jihui yoshin?

36. ¿Yoina yoshin?

37. ¿Huetsa yoshin: maya nihue, maca, nii, paro, tae, toro, yan?

38. ¿Caya copiaqui?

39. ¿Onanya, meraya, yobecan mia acari?

¿Quién te ha tratado de esta enfermedad?

44-50. ¿Médico, sanitario o promotor de salud, raomis, tobiamis, baquemis, onanya, meraya?

51. ¿Que rao te dio? Jlahue rao qui mia meniai?

67. Sintoma dominante.

68. Diagnóstico médico.

Este trabajo fué posible por un financiamiento de la Fundación B. Lelong del Centre National de la Recherche Scientifique. El señor Bernard LeLong era un gran conocedor de la Selva Peruana. 